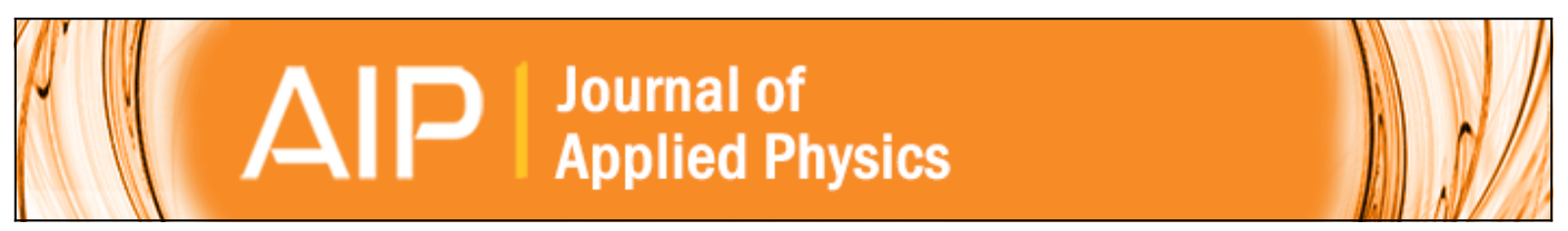

\title{
Transport on the surface of a topological insulator
}

V. Vargiamidis and P. Vasilopoulos

Citation: Journal of Applied Physics 116, 063713 (2014); doi: 10.1063/1.4892892

View online: http://dx.doi.org/10.1063/1.4892892

View Table of Contents: http://scitation.aip.org/content/aip/journal/jap/116/6?ver=pdfcov

Published by the AIP Publishing

\section{Articles you may be interested in}

Coulomb impurity scattering in topological insulator thin films

Appl. Phys. Lett. 105, 033118 (2014); 10.1063/1.4891574

Controllable fully spin-polarized transport in a ferromagnetically doped topological insulator junction

J. Appl. Phys. 115, 154310 (2014); 10.1063/1.4871683

Quantum magnetotransport properties of ultrathin topological insulator films

J. Appl. Phys. 113, 043720 (2013); 10.1063/1.4789626

Magnetically doped semiconducting topological insulators

J. Appl. Phys. 112, 063912 (2012); 10.1063/1.4754452

Surface versus bulk state in topological insulator $\mathrm{Bi} 2 \mathrm{Se} 3$ under environmental disorder

Appl. Phys. Lett. 99, 012109 (2011); 10.1063/1.3607484

\section{A|P| $\left.\right|_{\text {Applied Physics }} ^{\text {Journal of }}$}

Journal of Applied Physics is pleased to announce André Anders as its new Editor-in-Chief 


\title{
Transport on the surface of a topological insulator
}

\author{
V. Vargiamidis and P. Vasilopoulos \\ Department of Physics, Concordia University, 7141 Sherbrooke Ouest, Montréal, Québec H4B 1R6, Canada
}

(Received 24 June 2014; accepted 31 July 2014; published online 14 August 2014)

\begin{abstract}
We study theoretically dc and ac transport on the surface of a three-dimensional topological insulator when its time-reversal symmetry is broken. Starting with a Kubo formula, we derive an explicit expression for the dc Hall conductivity, valid for finite temperatures. At zero temperature this expression gives the dc half-quantum Hall conductivity, provided the Fermi level lies in the gap. Corrections when the Fermi level is outside the gap and scattering by impurities are quantified. The longitudinal conductivity is also examined. At finite frequencies, we find a modified Drude term in $\sigma_{x x}(\omega)$ and logarithmic, frequency-dependent corrections in $\sigma_{y x}(\omega)$. The ac Hall conductivity exhibits a robust logarithmic singularity for excitation energies equal to the gapwidth. For these energies, we also find that the power spectrum, which is pertinent to optical experiments, exhibits drastic increase. The Hall conductivity remains almost unaffected for temperatures up to approximately 300 K. (C) 2014 AIP Publishing LLC. [http://dx.doi.org/10.1063/1.4892892]
\end{abstract}

\section{INTRODUCTION}

A strong three-dimensional (3D) topological insulator (TI) is a novel quantum state of matter, which possesses surface conducting states in the bulk energy gap. ${ }^{1-7}$ The surface states are topologically protected and can be well described at low energies as helical Dirac fermions. Angle-resolved photoemission spectroscopy experiments confirmed the existence of Dirac-like surface states in $\mathrm{Bi}_{2} \mathrm{Se}_{3},{ }^{8} \mathrm{Bi}_{2} \mathrm{Te}_{3},{ }^{9}$ and $\mathrm{Sb}_{2} \mathrm{Te}_{3} .{ }^{9}$ The helical Dirac fermions in the surface states of a TI reveal a lot of unconventional properties such as the topological magnetoelectric effect ${ }^{10}$ and the image magnetic monopole effect. ${ }^{11}$

As far as transport phenomena are concerned, theoretical investigations have focused mostly on the effects of the characteristic spin-momentum locking of the helical TI surface states, which has an obvious potential in spintronics. ${ }^{12-16}$ There have been also studies of transport and optical properties of thin-film TIs. ${ }^{17,18}$ However, some topics have received only a limited attention, for instance, disorder, and ac transport.

The purpose of this paper is to study dc and ac transport on the surface of a 3D TI, when its time-reversal symmetry (TRS) is broken. Once a TRS-breaking perturbation is introduced, a gap is induced at the Dirac points of the TI, and the system enters a half-quantum Hall phase ${ }^{6}$ regardless of the detailed form of the perturbation. The surface half-quantum Hall effect is a unique property of the surface states of $3 \mathrm{D}$ TIs and has been discussed previously ${ }^{6,10}$ but has not been directly observed yet. In particular, when the Fermi level lies inside the gap, it has been predicted that the Hall conductivity is quantized as $\pm e^{2} /(2 h)$. Up to now though we are not aware of any explicit derivation of the surface half-quantum Hall effect and of any corrections to it resulting, e.g., from scattering. In this paper, besides providing an explicit derivation, we also quantify the corrections when the Fermi level is outside the gap and take into account weak, elastic scattering by impurities, for which the topological states are very robust. ${ }^{19}$ In addition, we study the longitudinal conductivity and its dependence on the carrier density and type of impurity potential. We also evaluate the finite-frequency conductivities $\sigma_{y x}(i \omega)$ and $\sigma_{x x}(i \omega)$ including scattering, and analyze the power spectrum which is relevant to optical experiments. Interestingly, we find a robust logarithmic singularity in the ac Hall conductivity when the photon energy reaches the gap energy. For this energy, the power spectrum exhibits sharp increase. For both dc and ac transport, we take into account finite temperatures. All calculations are carried out in the framework of linear response theory.

The rest of the paper is organized as follows. In Sec. II, we present one-electron properties and the relevant conductivities. In Sec. III, we discuss dc transport while ac transport and the power spectrum are discussed in Sec. IV. In Sec. V, we assess the influence of finite temperatures, and a summary of our results is presented in Sec. VI.

\section{FORMALISM}

\section{A. One-electron attributes}

The helical metallic surface states of a 3D TI are described by a Dirac-like Hamiltonian

$$
H_{s}(\mathbf{k})=\hbar v_{F}\left[\sigma_{x} k_{y}-\sigma_{y} k_{x}\right]+\Delta_{z} \sigma_{z},
$$

where $\sigma_{\mu}, \mu=x, y, z$ are the Pauli matrices that act on the spin degrees of freedom and $k_{x}$ and $k_{y}$ are the components of the electron's wave vector $\mathbf{k}$. The first term in the Hamiltonian captures the gapless Dirac-cone energy spectrum of the surface states. The influence of a TRS-breaking perturbation is captured by the mass term $\Delta_{z} \sigma_{z}$, which opens a gap at the Dirac point. An obvious physical source for the mass term is the Zeeman interaction between spins and an external magnetic field. Another possibility is to cover the surface of a 3D TI with magnetically ordered spins. 
In order to diagonalize $H_{s}(\mathbf{k})$, we first write $k_{ \pm}$ $= \pm i k e^{\mp i \varphi_{\mathbf{k}}}$, where $\tan \varphi_{\mathbf{k}}=k_{y} / k_{x}$ and $k=|\mathbf{k}|=\left(k_{x}^{2}+k_{y}^{2}\right)^{1 / \overline{2}}$. We also set $\epsilon_{\mathbf{k}}=\left(\Delta_{z}^{2}+\hbar^{2} v_{F}^{2} k^{2}\right)^{1 / 2}, \cos \theta=\Delta_{z} / \epsilon_{\mathbf{k}}$, and $\sin \theta$ $=\hbar v_{F} k / \epsilon_{\mathbf{k}}$. Then, Eq. (1) takes the form $H_{s}(\mathbf{k})=\epsilon_{\boldsymbol{k}} h(\mathbf{k})$ with

$$
h(\mathbf{k})=\left(\begin{array}{cc}
\cos \theta & \sin \theta e^{-i \varphi_{\mathbf{k}}^{\prime}} \\
\sin \theta e^{i \varphi_{\mathbf{k}}^{\prime}} & -\cos \theta
\end{array}\right),
$$

where $\varphi_{\mathbf{k}}^{\prime}=\varphi_{\mathbf{k}}-\pi / 2$. Since the matrix $h(\mathbf{k})$ is hermitian, it has real eigenvalues and its eigenfunctions, corresponding to different eigenvalues, are orthogonal. It is also unitary, i.e., its eigenvalues are of unit magnitude. They are $\lambda= \pm 1$, which entails that those of $H_{s}(\mathbf{k})$ are

$$
E_{\lambda, \mathbf{k}}=\lambda \epsilon_{\mathbf{k}}=\lambda \sqrt{\Delta_{z}^{2}+\hbar^{2} v_{F}^{2} k^{2}} .
$$

The index $\lambda$ represents the valence $(\lambda=-1)$ and conduction $(\lambda=+1)$ bands, respectively. The energy spectrum is plotted in Fig. 1 as a function of $\beta=\hbar v_{F} k /\left|\Delta_{z}\right|$ for $\Delta_{z}=5 \mathrm{meV}$ (solid curve). The dashed (red) curve is for $\Delta_{z}=0$. Note that the spectrum is the same as that of graphene in the presence of a mass term.

The eigenstates of $H_{s}(\mathbf{k})$ are two-component spinors and can be obtained following a standard diagonalization procedure. They are given by

$$
\Psi_{\lambda, \mathbf{k}}(\mathbf{r})=\frac{1}{\sqrt{2 S}}\left(\begin{array}{c}
\sqrt{1+\lambda \delta_{k}} \\
\lambda \sqrt{1-\lambda \delta_{k}} e^{i \varphi_{\mathbf{k}}^{\prime}}
\end{array}\right) e^{i \mathbf{k} \cdot \mathbf{r}},
$$

where $\delta_{k}=\Delta_{z} / \epsilon_{\mathbf{k}}$ and $S$ is the area of the of the TI. We notice that in the limit $\Delta_{z} \rightarrow 0$ we obtain the eigenstates in graphene if we consider only one of its two valleys. ${ }^{20}$

\section{Density of states}

An important property is the density of states $D(E)$, which is obtained from $D(E)=\sum_{\zeta} \delta\left(E_{\zeta}-E\right)$ with $|\zeta\rangle \equiv|\lambda, \mathbf{k}\rangle$. Replacing the $\delta$ function by a Lorentzian of width $\Gamma$, i.e., $\delta\left(E_{\lambda, \mathbf{k}}-E\right) \approx(\Gamma / \pi)\left(1 /\left[\left(E_{\lambda, \mathbf{k}}-E\right)^{2}+\Gamma^{2}\right]\right)$, and transforming the sum over $\mathbf{k}$ to an integral over the energy, we obtain

$$
D(E)=\frac{g|E|}{2 \pi^{2} \hbar^{2} v_{F}^{2}}\left[\pi-\tan ^{-1} E_{+}+\tan ^{-1} E_{-}+(\Gamma / 2|E|) \ln \left\{\left(1+E_{+}^{2}\right) /\left(1+E_{-}^{2}\right)\right\}\right] \Theta\left(|E|-\left|\Delta_{z}\right|\right),
$$

with $E_{ \pm}=\left(|E| \pm\left|\Delta_{z}\right|\right) / \Gamma$ and $\Theta(x)$ the Heaviside function. The factor $g$ takes into account the degeneracy due to internal degrees of freedom. In contrast to graphene, where $g=4$ due to the four Dirac cones $(2$ valley $\times 2$ spin), it turns out that $g=1$ on the surface of a TI. ${ }^{5,6}$ In fact, the number of Dirac cones is a key difference between the surface-state theory for 3D TIs and graphene or any 2D Dirac system. In the limit $\Gamma \rightarrow 0$, we obtain the simpler, unbroadened density of states $D(E)=g|E| /\left(2 \pi \hbar^{2} v_{F}^{2}\right)$ with $|E|>\left|\Delta_{z}\right|$. The density of states as a function of the energy is shown in Fig. 2 for $\Gamma=0$ (solid lines) and $\Gamma \neq 0$ (dashed, red lines). Notice that for $\left|\Delta_{z}\right| \rightarrow 0$, the density of states is identical in form to that

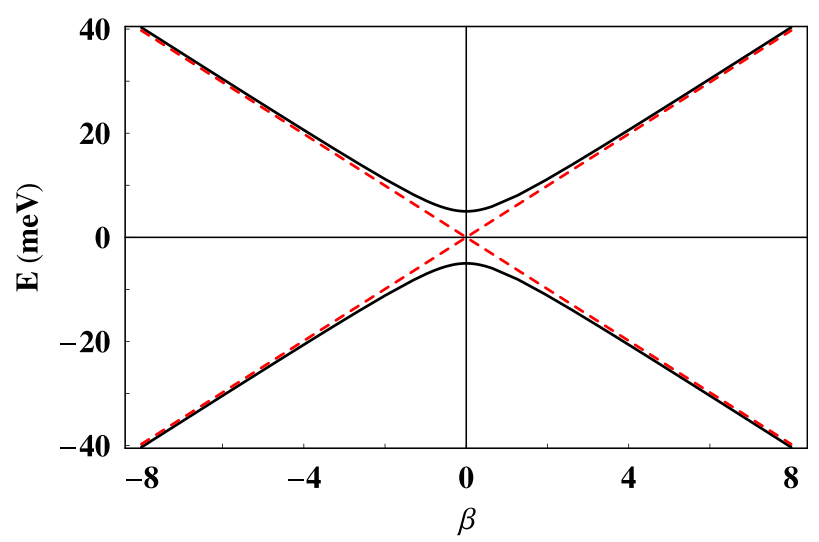

FIG. 1. Energy spectrum $E_{\lambda, \beta}$ as a function of the dimensionless parameter $\beta=\hbar v_{F} k /\left|\Delta_{z}\right|$ for $v_{F}=6.2 \times 10^{5} \mathrm{~m} / \mathrm{s}$ and $\Delta_{z}=5 \mathrm{meV}$. The dashed curve is for $\Delta_{z}=0$. in gapless graphene as shown in Fig. 2 by the (blue) longdashed lines. However, since valley and spin degeneracies are absent on the surface of a TI, the slope of $D(E)$ is only one fourth of the corresponding value of gapless graphene. We also note that the density of states is zero for energies smaller than the gap $2\left|\Delta_{z}\right|$ and experience a jump from zero to a finite value $\left(\left|\Delta_{z}\right| / 2 \pi \hbar^{2} v^{2}\right)$ at the energy $|E|=\left|\Delta_{z}\right|$.

\section{Matrix elements of the velocity operator}

In order to evaluate the various conductivities, we need the matrix elements of the velocity operator, i.e.,

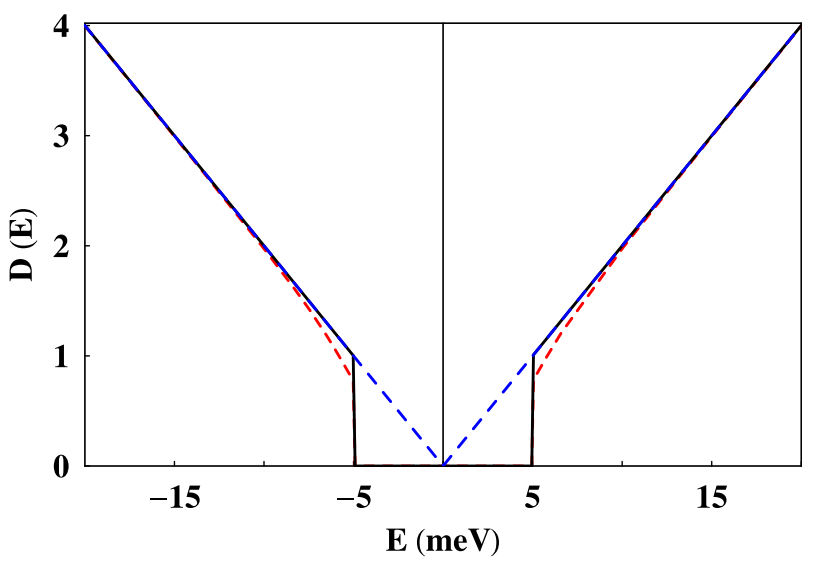

FIG. 2. Density of states $D(E)$ (in units of $1 / 2 \pi \hbar^{2} v_{F}^{2}$ ) as a function of the energy. The solid curve is for $\Gamma=0$ and the dashed (red) one for $\Gamma \neq 0$. The long-dashed (blue) curve is for $\Delta_{z}=0$. The values of the parameters are the same as in Fig. 1. 
$\left\langle\Psi_{\lambda, \mathbf{k}}\left|v_{\nu}\right| \Psi_{\lambda^{\prime}, \mathbf{k}^{\prime}}\right\rangle=v_{\nu, \zeta \zeta^{\prime}}$, where $\nu=x, y$, and $\zeta, \zeta^{\prime}$ stand for $(\lambda, \mathbf{k})$ and $\left(\lambda^{\prime}, \mathbf{k}^{\prime}\right)$, respectively. They are readily evaluated and read

$$
v_{x, \zeta \zeta^{\prime}}=\left(i v_{F} / 2\right) V_{-}, \quad v_{y, \zeta \zeta^{\prime}}=\left(v_{F} / 2\right) V_{+},
$$

where

$$
\begin{aligned}
V_{ \pm}= & {\left[\lambda^{\prime} \sqrt{\left(1+\lambda \delta_{k}\right)\left(1-\lambda^{\prime} \delta_{k}\right)} e^{i \varphi_{\mathbf{k}^{\prime}}}\right.} \\
& \left. \pm \lambda \sqrt{\left(1-\lambda \delta_{k}\right)\left(1+\lambda^{\prime} \delta_{k}\right)} e^{-i \varphi_{\mathbf{k}^{\prime}}}\right] \delta_{\mathbf{k k}^{\prime}} .
\end{aligned}
$$

\section{B. Linear-response conductivity expressions}

We adopt the formalism developed in Ref. 21. We consider a many-body system described by the Hamiltonian $H=H_{0}+H_{I}-\mathbf{R} \cdot \mathbf{F}(t)$, where $H_{0}$ is the unperturbed part, $H_{I}$ is a binary-type interaction (e.g., between electrons and impurities or phonons), and $-\mathbf{R} \cdot \mathbf{F}(t)$ is the interaction of the system with the external field $F(t)$. For conductivity problems $\mathbf{F}(t)=e \mathbf{E}(t)$, where $\mathbf{E}(t)$ is the electric field, e is the electron charge, $\mathbf{R}=\sum_{\mathbf{r}_{i}}$, and $\mathbf{r}_{i}$ is the position operator of electron i. In the representation in which $H_{0}$ is diagonal the many-body density operator $\rho=\rho^{d}+\rho^{\text {nd }}$ has a diagonal part $\rho^{d}$ and a nondiagonal part $\rho^{\text {nd }}$. Further, for weak electric fields and weak scattering potentials, for which the first Born approximation applies, the corresponding conductivity tensor has a diagonal part $\sigma_{\mu \nu}^{d}$ and a nondiagonal part $\sigma_{\mu \nu}^{n d}$, i.e., $\sigma_{\mu \nu}=\sigma_{\mu \nu}^{d}+\sigma_{\mu \nu}^{n d}$, where $\mu, \nu=x, y$.

Now, in general, there are two kinds of currents, diffusive and hopping, but usually only one of them is present. In this work, with no magnetic field present, there is only diffusive current since the hopping contribution vanishes identically, see Eq. (2.65) in Ref. 21. Then, for quasi-elastic scattering

$$
\sigma_{\mu \nu}^{d}(i \omega)=\frac{\beta e^{2}}{S} \sum_{\zeta} f_{\zeta}\left(1-f_{\zeta}\right) \frac{v_{\nu \zeta} v_{\mu \zeta} \tau_{\zeta}}{1+i \omega \tau_{\zeta}}
$$

where $\tau_{\zeta}$ is the momentum relaxation time, $\omega$ is the frequency, and $v_{\mu \zeta}=\left\langle\zeta\left|v_{\mu}\right| \zeta\right\rangle$ are the diagonal matrix elements of the velocity operator with $\mu=x, y$ and $|\zeta\rangle=\left|\lambda, k_{x}, k_{y}\right\rangle$. Further, $f_{\zeta}=\left[1+\exp \beta\left(E_{\zeta}-E_{F}\right)\right]^{-1}$ is the Fermi-Dirac distribution function with $\beta=1 / k_{B} T, T$ is the temperature, and $E_{F}$ is the Fermi level.

Regarding the contribution $\sigma_{\mu \nu}^{n d}$ one can use $\mathrm{e}^{22}$ the identity $f_{\zeta}\left(1-f_{\zeta^{\prime}}\right)\left[1-\exp \beta\left(E_{\zeta}-E_{\zeta^{\prime}}\right)\right]=f_{\zeta}-f_{\zeta^{\prime}}$ and cast the original form ${ }^{21}$ in the more familiar one

$$
\sigma_{\mu \nu}^{n d}(i \omega)=\frac{i \hbar e^{2}}{S} \sum_{\zeta \neq \zeta^{\prime}} \frac{\left(f_{\zeta}-f_{\zeta^{\prime}}\right) v_{\nu \zeta^{\prime}} v_{\mu \zeta^{\prime} \zeta}}{\left(E_{\zeta}-E_{\zeta^{\prime}}\right)\left(E_{\zeta}-E_{\zeta^{\prime}}+\hbar \omega+i \Gamma_{\zeta}\right)},
$$

where $v_{\nu \zeta^{\prime}}=\left\langle\zeta\left|v_{\nu}\right| \zeta^{\prime}\right\rangle$ and $v_{\mu \zeta^{\prime} \zeta}=\left\langle\zeta^{\prime}\left|v_{\nu}\right| \zeta\right\rangle$ are the nondiagonal matrix elements of the velocity operator. The sum runs over all quantum numbers $|\zeta\rangle=\left|\lambda, k_{x}, k_{y}\right\rangle$ and $\left|\zeta^{\prime}\right\rangle=$ $\left|\lambda^{\prime}, k_{x}^{\prime}, k_{y}^{\prime}\right\rangle$ provided $\zeta \neq \zeta^{\prime}$. The infinitesimal quantity $\epsilon$ in the original form ${ }^{21}$ has been replaced by $\Gamma_{\zeta}$ to account for the broadening of the energy levels.
It is convenient and rather instructive to first present the results for dc transport $(\omega=0)$ and then those for ac transport $(\omega \neq 0)$.

\section{DC TRANSPORT}

\section{A. Hall conductivity}

We consider now the dc Hall conductivity. We set $\sigma_{y x}^{n d}(0) \equiv \sigma_{y x}^{n d}$ in Eq. (9) and we consider first the case with no level broadening $\Gamma=0$. Because the matrix elements of the components $v_{x}$ and $v_{y}$ of the velocity operator $\mathbf{v}$ are diagonal in $\mathbf{k}$ the only elements that contribute to Eq. (9) are $v_{\mu,+,-}=\left\langle+, \mathbf{k}\left|v_{\mu}\right|-, \mathbf{k}^{\prime}\right\rangle$ and $v_{\mu,-,+}=\left\langle-, \mathbf{k}\left|v_{\mu}\right|+, \mathbf{k}^{\prime}\right\rangle$ and their complex conjugates. With the help of Eqs. (6) and (7), we obtain

$$
\begin{aligned}
& v_{x,+,-}=-\left(i v_{F} / k\right)\left(k_{y}-i \delta_{k} k_{x}\right), \\
& v_{y,+,-}=\left(i v_{F} / k\right)\left(k_{x}+i \delta_{k} k_{y}\right) .
\end{aligned}
$$

Substituting Eq. (10) in Eq. (9), we obtain

$$
\begin{aligned}
\sigma_{y x}^{n d}= & \left(i \hbar e^{2} / S\right) \sum_{\mathbf{k}}\left(f_{-, \mathbf{k}}-f_{+, \mathbf{k}}\right) / 4 \epsilon_{\mathbf{k}}^{2} \\
& \times\left[v_{x,-,+} v_{y,+,-}-v_{x,+,-,} v_{y,-,+}\right] \\
= & \left(2 \hbar v_{F}^{2} e^{2} \Delta_{z} / S\right) \sum_{\mathbf{k}}\left(f_{-, \mathbf{k}}-f_{+, \mathbf{k}}\right) / 4 \epsilon_{\mathbf{k}}^{3} .
\end{aligned}
$$

The sum over $\mathbf{k}$ is transformed to an integral, using the prescription $\sum_{\mathbf{k}} \rightarrow\left(S / 4 \pi^{2}\right) \int d^{2} k$, and the integral is carried out in polar coordinates. This gives the dc Hall conductivity for nonzero temperatures. For zero temperature and the Fermi level in the gap between $E_{-, \mathbf{k}}$ and $E_{+, \mathbf{k}}$, the Fermi functions reduce to $f_{-, \mathbf{k}}=1$ and $f_{+, \mathbf{k}}=0$. With $0 \leq k \leq \infty$ this gives the result of Ref. 6 ,

$$
\sigma_{y x}^{n d}=\frac{\Delta_{z}}{\left|\Delta_{z}\right|} \frac{e^{2}}{2 h}
$$

The derivation of the half-quantum Hall conductivity is valid if the continuum Hamiltonian in Eq. (1) applies. In particular, deviations from the Dirac fermion model at large momenta, which are necessary in the case of $2 \mathrm{D}$ systems, ${ }^{5,6}$ are not included in the above calculation. However, on the surface of a 3D TI such contributions from large momenta vanish, ${ }^{10}$ which makes them different from all 2D systems. Therefore, the half-quantum Hall effect is a unique property of the surface states of 3D TIs, which distinguishes them from all pure 2D systems.

When the Fermi level lies in the electron band, with $0 \leq k \leq k_{F}$ and for relatively low temperatures, we obtain

$$
\sigma_{y x}^{n d}=\frac{e^{2}}{2 h} \frac{\Delta_{z}}{E_{F}} \Theta\left(E_{F}-\Delta_{z}\right)
$$

with the $\Theta(\ldots)$ function reminding $E_{F} \geq \Delta_{z}$. Thus, when the Fermi level lies outside the gap, the Hall conductivity is not half-quantized anymore. We plot $\sigma_{y x}^{n d}$, given by Eq. (13), versus the (dimensionless) Fermi energy $\epsilon_{F}=E_{F} / \Delta_{z}$ in Fig. 3. As can be seen, for $\epsilon_{F}>1$, the Hall conductivity decreases 


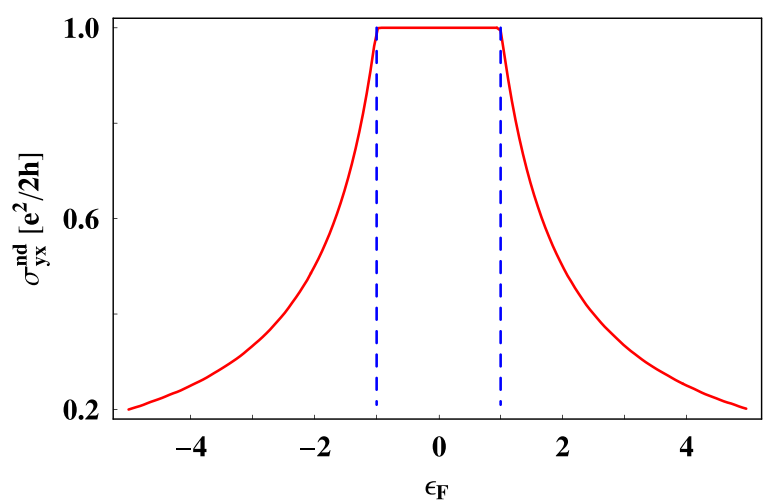

FIG. 3. Hall conductivity $\sigma_{y x}^{n d}$ as a function of the dimensionless Fermi energy $\epsilon_{F}=E_{F} / \Delta_{z}$. When $E_{F}$ lies in the gap between $-\left|\Delta_{z}\right|$ and $\left|\Delta_{z}\right|$, we have $\sigma_{y x}^{n d}=\left(\Delta_{z} /\left|\Delta_{z}\right|\right)\left(e^{2} / 2 h\right)$. For $E_{F}$ outside this gap, $\sigma_{y x}^{n d}$ decreases inversely proportional to $\epsilon_{F}$, see Eq. (13).

inversely proportional to $\epsilon_{F}$. We have verified by a numerical evaluation of Eq. (11) that the Hall conductivity is very robust against the temperature, as shown in Fig. 7 in Sec. V for $\alpha=0$. Similar result has been obtained in Ref. 23 for the case in which the mass is replaced by the strength of perpendicular magnetization.

We now consider the case with nonzero level broadening $(\Gamma \neq 0)$ and assume $\Gamma \ll E_{F}$. A straightforward calculation yields

$$
\sigma_{y x}^{n d}=\frac{i e^{2}}{2 h} \frac{\Delta_{z}}{\Gamma} \ln \left(\frac{1-i \gamma}{1+i \gamma}\right)=\frac{e^{2}}{2 h} \frac{\tan ^{-1} \gamma}{\gamma},
$$

where $\gamma=\Gamma / 2\left|\Delta_{z}\right|$ is the (dimensionless) level broadening. Expanding the logarithms for $\gamma<1$, we obtain

$$
\sigma_{y x}^{n d}=\frac{\Delta_{z}}{\left|\Delta_{z}\right|} \frac{e^{2}}{2 h}\left[1-\frac{\gamma^{2}}{3}+\frac{\gamma^{4}}{5}+\cdots\right] .
$$

It can be seen from Eq. (15) that the leading-order correction to the usual result is $\gamma^{2} / 3$.

We now turn to the component $\sigma_{x x}^{n d}$, which is evaluated in the same manner as $\sigma_{y x}^{n d}$. If we neglect $\Gamma$, we find that $\sigma_{x x}^{n d}$ vanishes identically. If we do not neglect $\Gamma$ the result is very unwieldy but very small and therefore it will be neglected.

\section{B. Longitudinal conductivity}

We consider now the diagonal components $\sigma_{y x}^{d}$ and $\sigma_{x x}^{d}$ which are given by Eq. (8) for $\omega=0$. The component $\sigma_{y x}^{d}$ vanishes identically because $\left\langle\zeta\left|\mathbf{r}_{\mu}\right| \zeta\right\rangle=0$. As for $\sigma_{x x}^{d}$, using $\left\langle\lambda, \mathbf{k}\left|v_{\mu}\right| \lambda, \mathbf{k}\right\rangle=\lambda \hbar k_{\mu} v_{F}^{2} / \epsilon_{\mathbf{k}}, \mu=x, y$, we obtain

$$
\sigma_{x x}^{d}=\frac{\beta \hbar^{2} v_{F}^{4} e^{2}}{S} \sum_{\lambda \mathbf{k}}\left[k_{x}^{2} / \epsilon_{\mathbf{k}}^{2}\right] f_{\lambda, \mathbf{k}}\left(1-f_{\lambda, \mathbf{k}}\right) \tau_{\lambda, \mathbf{k}} .
$$

In general, the integral over $k$ can be evaluated numerically. However, we can obtain an approximate analytic result by assuming a constant relaxation time $\tau_{+, \mathbf{k}} \approx \tau_{-, \mathbf{k}} \approx \tau_{F}$ and consider very low temperatures for which the approximation $\beta f_{+, \mathbf{k}}\left(1-f_{+, \mathbf{k}}\right) \approx \delta\left(E_{+, \mathbf{k}}-E_{F}\right)$ holds. After integration over $\mathbf{k}$, we obtain

$$
\sigma_{x x}^{d} \approx \frac{\mathrm{e}^{2}}{2 h} \frac{\tau_{F}\left(E_{F}^{2}-\Delta_{z}^{2}\right)}{\hbar E_{F}} \Theta\left(E_{F}-\Delta_{z}\right)
$$

The relaxation time is calculated in the Appendix for shortrange and long-range impurity scattering. We also notice that in the limit $\Delta_{z} \rightarrow 0$, Eq. (17) agrees with the result obtained in Ref. 24 to within a factor of 2.

For finite $\Gamma$ and very low temperatures, we make the same approximations as above. Even with a constant relaxation time the result is unwieldy. However, for $\Gamma \ll E_{F}$ it is given by

$$
\begin{aligned}
\sigma_{x x}^{d} \approx & \frac{\mathrm{e}^{2}}{2 h} \frac{\tau_{F}\left(E_{F}^{2}-\Delta_{z}^{2}\right)}{\pi \hbar E_{F}}\left[\frac{\pi}{2}+\tan ^{-1} \frac{E_{F}-\Delta_{z}}{\Gamma}\right] \\
& \times \Theta\left(E_{F}-\Delta_{z}\right) .
\end{aligned}
$$

As can be easily verified, Eq. (18) reduces to Eq. (17) in the limit $\Gamma \rightarrow 0$.

It is appropriate to consider the dependence of $\sigma_{x x}^{d}$ on the carrier density $n_{e}$, through that on $k_{F}$, and the impurity density $n_{i}$ through that of $\tau$, cf. Eq. (A1). In a 2D system, surface roughness may give rise to short-range scattering. ${ }^{25}$ The corresponding rate $1 / \tau$ is given in Eq. (A3) for $\Delta_{z}=0$ and leads to a constant $\sigma_{x x}^{d}{ }^{25}$ The situation is different for longrange Coulomb potentials. For charged impurities screened by the conduction electrons, we may identify $k_{s}$ (see the Appendix) with the Thomas-Fermi wave vector

$$
k_{s}=\left(2 \pi e^{2} / \epsilon\right) D\left(E_{F}\right),
$$

where $\epsilon$ is the relative dielectric constant and $D\left(E_{F}\right)$ is the density of states at the Fermi level. With the help of Eq. (A4), we plot $\sigma_{x x}^{d}$ in Fig. 4 versus the ratio $n_{e} / n_{i}$ of the carrier density to the impurity density for $\epsilon=100$, a value appropriate to $\mathrm{Bi}_{2} \mathrm{Se}_{3}$, and $\epsilon=80$, with $n_{i}=4 \times 10^{13} \mathrm{~cm}^{-2}$ and $\hbar v_{F}=4.1 \mathrm{eV} \AA$. Note that $\sigma_{x x}^{d}$ increases linearly with the carrier density. This is also the case for $\Delta_{z}=0$ (Ref. 7) and that of graphene. ${ }^{26}$ However, the large value of the dielectric constant implies a large reduction in the Coulomb potential yielding a smaller scattering rate which can account for the faster increase of the conductivity in TIs than in graphene.

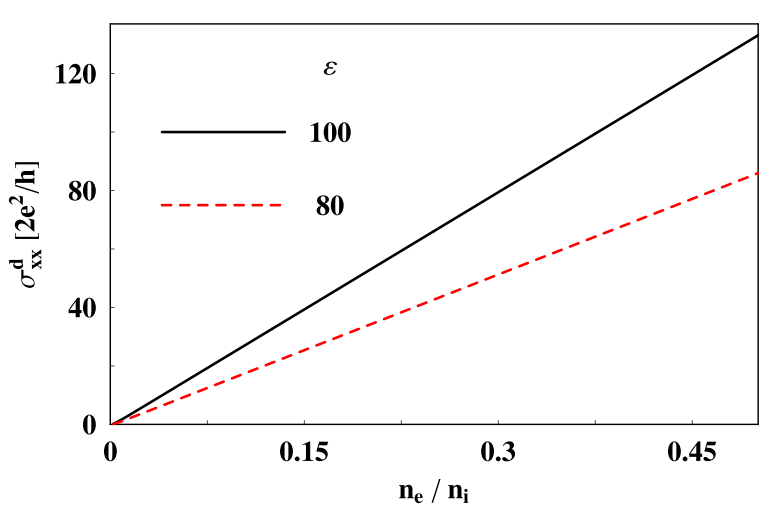

FIG. 4. Conductivity $\sigma_{x x}^{d}$ for screened Coulomb scatterers as a function of the surface carrier density $n_{e}$ for $\epsilon=100$ (solid curve) and $\epsilon=80$ (solid curve). Both curves are for $n_{i}=4 \times 10^{13} \mathrm{~cm}^{-2}$ and $\hbar v_{F}=4.1 \mathrm{eV} \AA$. 


\section{AC TRANSPORT}

\section{A. Conductivities}

We now consider the finite-frequency conductivity $\sigma_{y x}^{n d}(i \omega)$, which is given by Eq. (9), and its evaluation follows that of $\sigma_{y x}^{n d}(0)$. With the help of the matrix elements in Eqs.

(6) and (7), we can express Eq. (9) as

$$
\begin{aligned}
\sigma_{y x}^{n d}(i \omega)= & \left(2 \hbar v^{2} e^{2} \Delta_{z} / S\right) \sum_{\mathbf{k}}\left(f_{-, \mathbf{k}}-f_{+, \mathbf{k}}\right) / 4 \epsilon_{\mathbf{k}}^{2} \\
& \times\left[\frac{2 \epsilon_{\mathbf{k}}+\hbar \omega+i \Gamma}{\left(2 \epsilon_{\mathbf{k}}+\hbar \omega\right)^{2}+\Gamma^{2}}+\frac{2 \epsilon_{\mathbf{k}}-\hbar \omega-i \Gamma}{\left(2 \epsilon_{\mathbf{k}}-\hbar \omega\right)^{2}+\Gamma^{2}}\right] .
\end{aligned}
$$

The sum over $\mathbf{k}$ is converted to an integral and the integral is carried out in polar coordinates with $0 \leq k \leq k_{F}$. In addition, we remark that $\hbar \omega$ and $\Gamma$ are at least one order of magnitude smaller than $E_{F}$, i.e., $\hbar \omega \ll E_{F}$ and $\Gamma \ll E_{F}$. Accordingly, terms of order $\Gamma / E_{F}$ and $\hbar \omega / E_{F}$ can be safely neglected. At zero temperature and with $E_{F}$ in the gap between $E_{-, \mathbf{k}}$ and $E_{+, \mathbf{k}}$, we obtain

$$
\sigma_{y x}^{n d}(i \omega)=\frac{\Delta_{z}}{\left|\Delta_{z}\right|} \frac{\mathrm{e}^{2}}{4 h}\left(\frac{\alpha+i \gamma}{\alpha^{2}+\gamma^{2}}\right) \ln \left(\frac{1+\alpha-i \gamma}{1-\alpha+i \gamma}\right),
$$

where $\alpha=\hbar \omega / 2\left|\Delta_{z}\right|$. Note that in the limit $\alpha \rightarrow 0$ Eq. (21) reduces to Eq. (14). We also notice that in the limit $\gamma \rightarrow 0$, the real part of $\sigma_{y x}^{n d}(i \omega)$ has a logarithmic divergence for $\alpha=1$, i.e., for frequency such that the photon energy equals the width of the gap $\hbar \omega=2\left|\Delta_{z}\right|$. The corresponding peak though is broadened with increasing $\Gamma$, as shown in Fig. 5, where we plot separately the real (upper) and imaginary (lower) parts of $\sigma_{y x}^{n d}(i \omega)$. The imaginary part influences the behaviour of the power spectrum, as discussed in Sec. IV B. The appearance of the singularity for $\alpha=1$ is related to the onset of particle-hole excitations ${ }^{27}$ for $\hbar \omega>2\left|\Delta_{z}\right|$; namely, for $\hbar \omega>2\left|\Delta_{z}\right|$, the electrons can absorb photons from the external ac field and create particle-hole pairs, whereas this effect is forbidden for $\hbar \omega<2\left|\Delta_{z}\right|$.

The logarithmic singularity in the real part of $\sigma_{y x}^{n d}(i \omega)$ is very robust over a wide range of temperatures as discussed in Sec. V. Its position is determined only by the width of the gap. This is remarkable because it means that the singularity

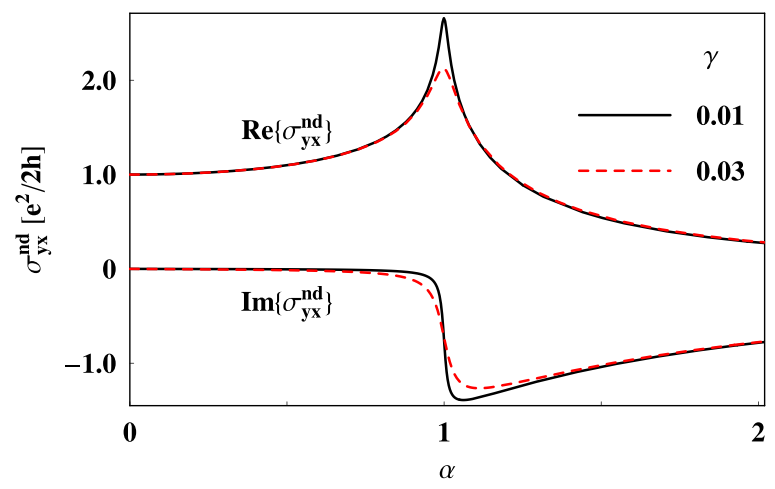

FIG. 5. The real (upper) and imaginary (lower) part of $\sigma_{y x}^{n d}(i \omega)$ vs $\alpha=$ $\hbar \omega / 2\left|\Delta_{z}\right|$ in units of $e^{2} / 2 h$. The solid (dashed) curves are for $\gamma=0.01$ $(\gamma=0.03), \gamma=\Gamma / 2\left|\Delta_{z}\right|$, and $\Delta_{z}>0$. Notice the dc limit $\sigma_{y x}^{n d}(0)=e^{2} / 2 h$. can be useful for the experimental determination of the gap in TIs by studying the ac Hall conductivity.

We further notice that for $\gamma=0$, Eq. (21) agrees with the recent result of Ref. 27, as well as that of Ref. 28, and is referred to as the optical Hall conductivity. However, for $\gamma \neq 0$ only numerical results were given in Refs. 27 and 28 .

We now consider the component $\sigma_{x x}^{n d}(i \omega)$. Neglecting $\Gamma$ a straightforward calculation yields

$$
\sigma_{x x}^{n d}(i \omega)=\frac{i \mathrm{e}^{2}}{2 h}\left[\frac{1}{2 \alpha}+\left(\frac{1}{4}+\frac{1}{4 \alpha^{2}}\right) \ln \left(\frac{1-\alpha}{1+\alpha}\right)\right] .
$$

Evidently this result diverges in the limit $\alpha \rightarrow 1$ or $\hbar \omega \rightarrow$ $2\left|\Delta_{z}\right|$ and was also noticed in Ref. 28. For $\hbar \omega \ll 2\left|\Delta_{z}\right|$, we can expand the logarithms and obtain, to leading order in $\alpha$, the simple result

$$
\sigma_{x x}^{n d}(i \omega)=-\frac{i \mathrm{e}^{2}}{h} \frac{\hbar \omega}{8\left|\Delta_{z}\right|}
$$

The evaluation of the component $\sigma_{x x}^{d}(i \omega)$ for $\Gamma=0$ follows verbatim that of $\sigma_{x x}^{d}(0)$ and the approximations stated after Eq. (16). Corresponding to Eq. (17), we obtain

$$
\sigma_{x x}^{d}(i \omega)=\frac{\mathrm{e}^{2}}{2 h} \frac{\tau_{F}}{1+i \omega \tau_{F}} \frac{E_{F}^{2}-\Delta_{z}^{2}}{\hbar E_{F}} \Theta\left(E_{F}-\Delta_{z}\right) \Theta\left(\hbar \omega-2\left|\Delta_{z}\right|\right) .
$$

The result (24) is similar to the semiclassical one of Ref. 28 that is valid only in the collisionless limit.

Now for $\Gamma \neq 0$ and very low temperatures, we make the replacement $\delta\left(E_{+, \mathbf{k}}-E_{F}\right) \approx(\Gamma / \pi)\left(1 /\left[\left(E_{+, \mathbf{k}}-E_{F}\right)^{2}+\Gamma^{2}\right]\right)$. With a constant relaxation time and $\Gamma \ll E_{F}$, the result for $\sigma_{x x}^{d}(i \omega)$ is given by Eq. (18) with $\tau_{F}$ replaced by $\tau_{F} /$ $\left(1+i \omega \tau_{F}\right)$.

\section{B. Power spectrum}

One important issue is the frequency dependence of the power absorbed in TIs, which is relevant to optical experiments. In our discussion below, we focus on the righthanded circularly polarized light.

Within linear response theory, the average power absorbed from light of frequency $\omega$ and electric field strength $E$ is given by

$$
P(\omega)=\frac{1}{2} E^{2} \operatorname{Re}\left\{\sigma_{x x}(i \omega)+\sigma_{y y}(i \omega)+i \sigma_{y x}(i \omega)-i \sigma_{x y}(i \omega)\right\} .
$$

We remark that $\sigma_{y y}(i \omega)=\sigma_{x x}(i \omega)$ and $\sigma_{y x}^{d}(i \omega)$ $=-\sigma_{x y}^{d}(i \omega)=0$. Also, $\sigma_{x y}^{n d}(i \omega)=-\sigma_{y x}^{n d}(i \omega)$. Then, Eq. (25) takes the form

$$
P(\omega)=E^{2} \operatorname{Re}\left\{\sigma_{x x}^{d}(i \omega)+\sigma_{x x}^{n d}(i \omega)+i \sigma_{y x}^{n d}(i \omega)\right\} .
$$

We show $P(\omega)$ in Fig. 6 versus $\alpha$ for two values of the broadening $\gamma$. The power spectrum vanishes for $\hbar \omega<2\left|\Delta_{z}\right|$, but we notice a sharp onset for excitation energies equal to the gapwidth of the TI, i.e., for $\hbar \omega \rightarrow 2\left|\Delta_{z}\right|$. However, the sharp 


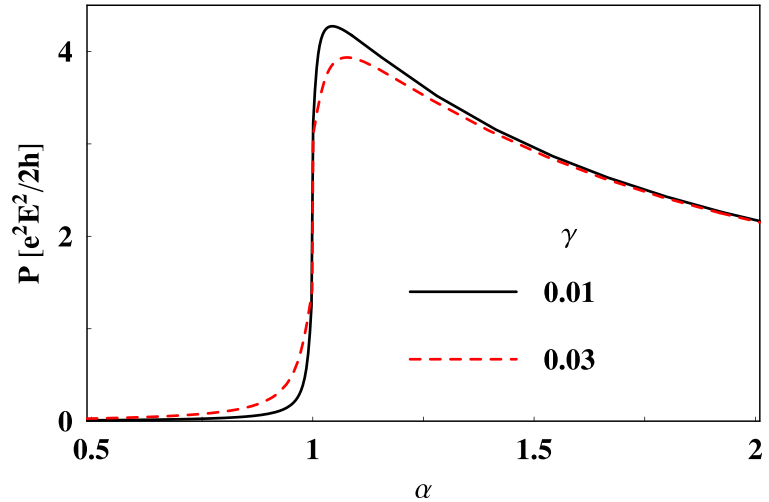

FIG. 6. Power spectrum vs $\alpha=\hbar \omega / 2\left|\Delta_{z}\right|$ in units of $E^{2} e^{2} / 2 h$. A relaxation time $\tau=7 \times 10^{-14} \mathrm{~s}$, a Fermi level $E_{F}=100 \mathrm{meV}$, and a mass term $\Delta_{z}=0.2 E_{F}$ were assumed. The curves are marked as in Fig. 5. The drastic increase for $\gamma \rightarrow 0$ weakens as $\gamma$ increases.

increase of the power spectrum for $\gamma \rightarrow 0$ is smeared out as $\gamma$ increases.

\section{FINITE TEMPERATURES}

We consider now the influence of finite temperatures. The real part of $\sigma_{y x}^{n d}(i \omega)$ in Eq. (20) is evaluated numerically and plotted in Fig. 7(a) as a function of $\alpha$, for increasing values of temperature. The Fermi energy is $E_{F}=0.1 \mathrm{eV}$, while $\Delta_{z}=0.012 \mathrm{eV}$ and $\Gamma=0.001 \mathrm{eV}$. We notice that the properties of the ac Hall conductivity do not change over a wide range of temperatures and the logarithmic singularity is robust. This effect can be used to determine the gap within a transport measurement. The overall behaviour is similar to that in Ref. 27, where even higher temperatures were considered, and rests on the tacit assumption that at these elevated temperatures other factors, e.g., phonons, are unable to significantly weaken this divergence or put into question the applicability of the formalism. In our case such factors are somewhat taken into account through the use of $\gamma$.

In Fig. 7(b), the real part of $\sigma_{y x}^{n d}(i \omega)$ is plotted as a function of temperature for two values of the frequency. The rest of the parameters have the same values as in Fig. 7(a). Note that both $\mathrm{dc}(\alpha=0)$ and ac $(\alpha=0.3)$ Hall conductivities remain unaffected with increasing temperature up to $T \approx 300 \mathrm{~K}$

\section{SUMMARY}

Summarizing, we have systematically studied dc and ac transport on the surface of a TI with TRS-breaking perturbation represented by a mass term $\Delta_{z}$, which opens a gap $2\left|\Delta_{z}\right|$ at the Dirac point. At zero temperature, we obtained the dc half-quantum Hall conductivity, $\sigma_{y x}=\left(\Delta_{z} /\left|\Delta_{z}\right|\right)\left(e^{2} / 2 h\right)$, provided the Fermi level $E_{F}$ lies in the gap and corrections due to scattering by impurities, which to our knowledge are new. We also obtained the expression $\sigma_{y x}=\left(\Delta_{z} / E_{F}\right)\left(e^{2} / 2 h\right)$ when the Fermi level lies in the conduction band. In addition, the longitudinal conductivity, for scattering by screened Coulomb impurities, was shown to depend linearly on the carrier density.

For ac transport, we obtained a modified Drude term in $\sigma_{x x}(\omega)$ and logarithmic, frequency-dependent corrections in
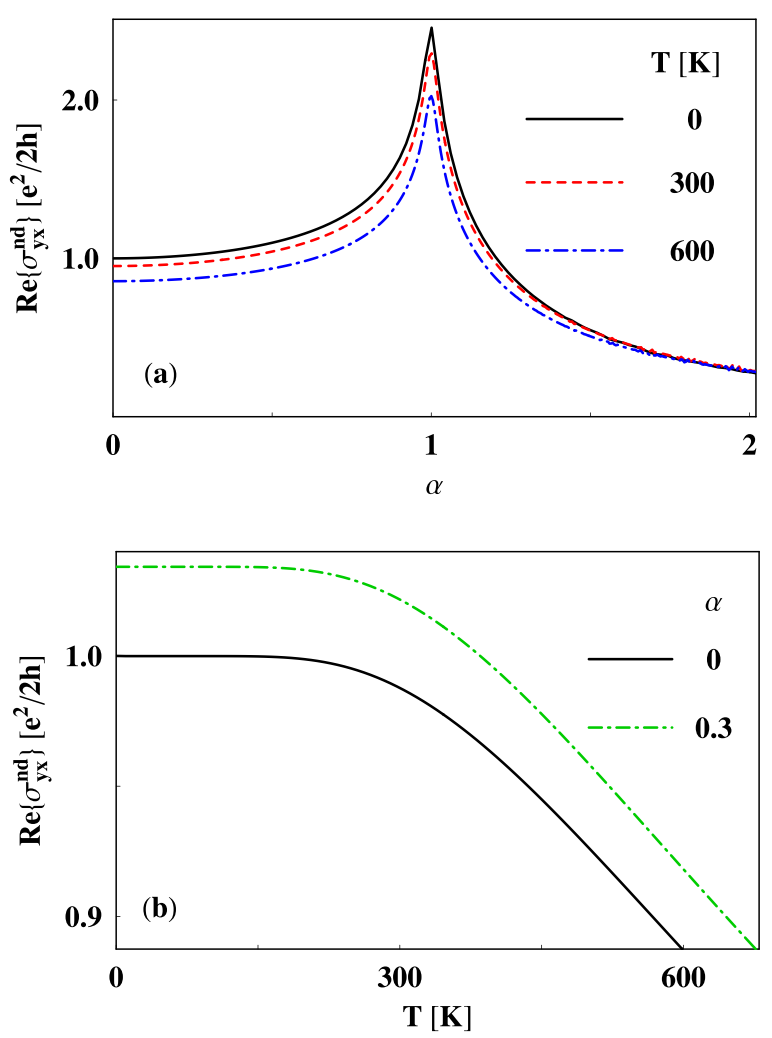

FIG. 7. (a) Real part of $\sigma_{y x}^{n d}(i \omega)$ as a function of $\alpha=\hbar \omega / 2\left|\Delta_{z}\right|$, in units of $e^{2} / 2 h$, for increasing values of temperature. (b) Real part of $\sigma_{y x}^{n d}(i \omega)$ as a function of temperature $T$ for zero (solid curve) and finite (dashed-dotted curve) frequencies.

$\sigma_{y x}(\omega)$. Interestingly, there is a logarithmic singularity in the ac Hall conductivity when the photon energy equals the width of the gap of the TI. This singularity survives over a wide range of temperatures and could be used to measure experimentally the gap. We also studied the power absorption spectrum, pertinent to optical experiments, and showed that it increases drastically for $\hbar \omega \rightarrow 2\left|\Delta_{z}\right|$. This last finding could be tested experimentally and help in the identification or observation of the ac half-quantum Hall conductivity. ${ }^{29}$

We also assessed the influence of finite temperatures and showed that the optical Hall conductivity and its peak, as well as its dc limit, remain almost unaffected up to approximately $300 \mathrm{~K}$.

\section{ACKNOWLEDGMENTS}

Our work was supported by the Canadian NSERC Grant No. OGP0121756.

\section{APPENDIX: RELAXATION TIME}

Below we briefly present the results for the relaxation rate $1 / \tau$ assuming elastic scattering by short-range and longrange impurities. Within the first Born approximation the standard formula takes the form

$$
\begin{aligned}
1 / \tau_{\zeta} \equiv & 1 / \tau_{\lambda k}=(2 \pi / \hbar) n_{i} \\
& \times \sum_{\lambda^{\prime} k^{\prime}}\left|\left\langle\lambda \mathbf{k}|U(\mathbf{r})| \lambda^{\prime} \mathbf{k}^{\prime}\right\rangle\right|^{2} \delta\left(E_{\lambda \mathbf{k}}-E_{\lambda^{\prime} \mathbf{k}^{\prime}}\right)(1-\cos \theta),
\end{aligned}
$$


where $U(\mathbf{r})$ is the impurity potential, $n_{i}$ is the impurity density, and $\theta=\theta_{\mathbf{k}, \mathbf{k}^{\prime}}$ is the angle between the initial $\mathbf{k}$ and final $\mathbf{k}^{\prime}$ wave vectors.

Since the scattering is elastic, we have $\lambda^{\prime}=\lambda$. A direct evaluation then gives the intermediate result

$$
\begin{aligned}
\frac{1}{\tau_{\lambda k}}= & \frac{n_{i}}{2 h}\left(\left[\Delta_{z}^{2}+\hbar^{2} v_{F}^{2} k^{2}\right]^{1 / 2} / \hbar^{2} v_{F}^{2}\right) \\
& \times \int\left[1+\cos \theta+\delta_{k}^{2}(1-\cos \theta)\right]|V(q)|^{2}(1-\cos \theta) d \theta,
\end{aligned}
$$

where $V(q)$ is the Fourier transform of $U(\mathbf{r})$, evaluated at $q=\left|\mathbf{k}-\mathbf{k}^{\prime}\right|=2 k \sin (\theta / 2)$. Notice that, for $\Delta_{z} \propto \delta_{k} \rightarrow 0$, the integrand takes the form $\left(1-\cos ^{2} \theta\right)|V(q)|^{2}$ and vanishes for small- and long-angle scattering as in the case of graphene without any mass term. ${ }^{26}$

(i) Short-range impurities. We have $U(\mathbf{r})=V_{0} \delta\left(\mathbf{r}-\mathbf{r}_{\mathbf{i}}\right)$ with $\mathbf{r}, \mathbf{r}_{\mathbf{i}}$ the position vectors of an electron and an impurity, respectively, and $V_{0}$ the constant strength of the potential. In this case, $V(q)=V_{0}$ and the final result is

$$
\frac{1}{\tau_{\lambda k}}=\frac{n_{i}}{2 h} \frac{V_{0}^{2}}{\hbar^{2} v_{F}^{2}} \frac{4 \Delta_{z}^{2}+\hbar^{2} v_{F}^{2} k^{2}}{\sqrt{\Delta_{z}^{2}+\hbar^{2} v_{F}^{2} k^{2}}} .
$$

For $\Delta_{z}=0$, this results agrees with that for graphene given in Ref. 26.

(ii) Long-range impurities. We assume a screened Coulomb potential $U(\mathbf{r})=e Q e^{-k_{s} r} / 4 \pi \epsilon_{0} \epsilon r$, where $k_{s}$ is the screening wave vector, $Q$ is the charge of the impurity, and $\epsilon$ is the dielectric constant. In this case, $|V(q)|^{2}=4 \pi^{2} U_{0}^{2} /\left(q^{2}+k_{s}^{2}\right)$ with $q=2 k \sin (\theta / 2)$ and $U_{0}=e Q / 4 \pi \epsilon_{0} \epsilon$. Again for $\Delta_{z} \propto \delta_{k} \rightarrow 0$, the integrand vanishes for small- and long-angle scattering. The integral in Eq. (A2) is evaluated by contour integration. With $s=k_{s}^{2} / 2 k_{F}^{2}$ the result for $1 / \tau_{\lambda k}$ is

$$
\begin{aligned}
\frac{1}{\tau_{\lambda k}}= & \frac{n_{i}}{2 h} \frac{2 \pi^{3} U_{0}^{2}}{\hbar^{2} v_{F}^{2} k_{F}^{2}}\left[\left(1+\delta_{k}^{2}\right)(1-1 / \sqrt{1+2 / s})\right. \\
& \left.+\left(1-\delta_{k}^{2}\right)[s-(1+s) / \sqrt{1+2 / s}]\right] .
\end{aligned}
$$

${ }^{1}$ B. A. Bernevig, T. L. Hughes, and S.-C. Zhang, Science 314, 1757 (2006). ${ }^{2}$ L. Fu, C. L. Kane, and E. J. Mele, Phys. Rev. Lett. 98, 106803 (2007).

${ }^{3}$ J. E. Moore and L. Balents, Phys. Rev. B 75, 121306(R) (2007).

${ }^{4}$ R. Roy, Phys. Rev. B 79, 195322 (2009).

${ }^{5}$ M. Z. Hasan and C. L. Kane, Rev. Mod. Phys. 82, 3045 (2010).

${ }^{6}$ X.-L. Qi and S.-C. Zhang, Rev. Mod. Phys. 83, 1057 (2011).

${ }^{7}$ D. Culcer, Physica E 44, 860 (2012).

${ }^{8}$ D. Hsieh, Y. Xia, D. Qian, L. Wray, J. H. Dil, F. Meier, J. Osterwalder, L. Patthey, J. G. Checkelsky, N. P. Ong, A. V. Fedorov, H. Lin, A. Bansil, D. Grauer, Y. S. Hor, R. J. Cava, and M. Z. Hasan, Nature (London) 460, 1101 (2009).

${ }^{9}$ D. Hsieh, Y. Xia, D. Qian, L. Wray, F. Meier, J. H. Dil, J. Osterwalder, L. Patthey, A. V. Fedorov, H. Lin, A. Bansil, D. Grauer, Y. S. Hor, R. J. Cava, and M. Z. Hasan, Phys. Rev. Lett. 103, 146401 (2009).

${ }^{10}$ X. L. Qi, T. L. Hughes, and S. C. Zhang, Phys. Rev. B 78, 195424 (2008); K. Nomura and N. Nagaosa, Phys. Rev. Lett. 106, 166802 (2011).

${ }^{11}$ X. L. Qi, R. Li, J. Zang, and S. C. Zhang, Science 323, 1184 (2009).

${ }^{12}$ S. Raghu, S. B. Chung, X.-L. Qi, and S.-C. Zhang, Phys. Rev. Lett. 104, 116401 (2010).

${ }^{13}$ S. Mondal, D. Sen, K. Sengupta, and R. Shankar, Phys. Rev. Lett. 104, 046403 (2010).

${ }^{14}$ T. Yokoyama, Y. Tanaka, and N. Nagaosa, Phys. Rev. B 81, 121401(R) (2010).

${ }^{15}$ D. Pesin and A. H. MacDonald, Nat. Mater. 11, 409 (2012).

${ }^{16} \mathrm{~T}$. Yokoyama and S. Murakami, Physica E 55, 1 (2014).

${ }^{17}$ H.-Z. Lu, W.-Y. Shan, W. Yao, Q. Niu, and S.-Q. Shen, Phys. Rev. B 81, 115407 (2010).

${ }^{18}$ A. A. Zyuzin and A. A. Burkov, Phys. Rev. B 83, 195413 (2011).

${ }^{19}$ X.-F. Wang, Y. Hu, and H. Guo, Phys. Rev. B 85, 241402(R) (2012).

${ }^{20}$ M. O. Goerbig, Rev. Mod. Phys. 83, 1193 (2011).

${ }^{21}$ M. Charbonneau, K. M. Van Vliet, and P. Vasilopoulos, J. Math. Phys. 23, 318 (1982).

${ }^{22}$ P. Vasilopoulos, Phys. Rev. B 32, 771 (1985).

${ }^{23}$ J. Zang and N. Nagaosa, Phys. Rev. B 81, 245125 (2010).

${ }^{24}$ B. Skinner and B. I. Shklovskii, Phys. Rev. B 87, 075454 (2013).

${ }^{25}$ T. Ando, A. B. Fowler, and F. Stern, Rev. Mod. Phys. 54, 437 (1982).

${ }^{26}$ T. Stauber, N. M. R. Peres, and F. Guinea, Phys. Rev. B 76, 205423 (2007); K. Nomura and A. H. MacDonald, Phys. Rev. Lett. 96, 256602 (2006).

${ }^{27}$ A. Hill, A. Sinner, and K. Ziegler, Eur. Phys. J. B 86, 53 (2013).

${ }^{28}$ W.-K. Tse and A. H. MacDonald, Phys. Rev. Lett. 105, 057401 (2010).

${ }^{29}$ The optical response to an ac signal, in the presence of magnetic field $B$, was discussed in Phys. Rev. Lett. 105, 166803 (2010), and in Refs. 27 and 28 for $B=0$; however, the power spectrum was not discussed at all, see also Sec. IV. 